\title{
Axillary reverse mapping in breast cancer surgery - functional study
}

\author{
David PAVLISTA ${ }^{1, *}$, Lukas DOSTALEK², Zuzana VELENSKA ${ }^{2}$ \\ ${ }^{1}$ Gynecologic Oncology Center, Department of Obstetrics and Gynecology, First Faculty of Medicine, General University Hospital in Prague, \\ Charles University in Prague, Prague, Czech Republic; ${ }^{2}$ Institute of Pathology, First Faculty of Medicine, General University Hospital in Prague, \\ Charles University in Prague, Prague, Czech Republic
}

${ }^{*}$ Correspondence: david.pavlista@vfn.cz

Received June 22, 2021 / Accepted September 1, 2021

\begin{abstract}
The aim of the study was to map the lymphatic drainage of the upper extremity that traverses the axilla and elucidate its relationship with the lymphatic drainage of the breast. In 79 breast cancer patients indicated to the axillary lymph node dissection for category cN1, cN2, Technetium-99m (particle size $<80 \mathrm{~nm}$ ) was applied prior to surgery at two injection sites between the second and third metacarpophalangeal joints to visualize upper extremity lymphatics. During the surgery, the axilla was anatomically divided into 6 quadrants. A C-Trak ${ }^{\oplus}$ device was used for the intraoperative detection of radioactivity. After verifying activity, the nodes were resected and their position was recorded. Active nodes were sent separately according to topographic localizations for microscopic examination. All affected nodes (both macro- and micrometastases) were recorded as positive. The location, involvement and radioactivity, and the number of lymph nodes obtained were analyzed. In total, 1,109 lymph nodes were removed and examined. Radioactive nodes were found in all 79 patients. A total of 230 radioactive nodes were found. 21 nodes were both radioactive and metastatically affected. Results show that part of the lymph from the upper extremity flows through the nodes in the central part of the axilla and mixes with the lymph from the breast. This suggests that lymphatic drainage of the upper limb cannot be functionally separated from lymphatic drainage of the breast. The results also explain the possible mechanical cause of arm lymphedema after sentinel lymph node biopsy.
\end{abstract}

Key words: breast cancer, lymphatic mapping, surgery, lymphedema

Based on the results of meta-analyses, surgical procedures are rationalized and minimized to reduce the number and severity of subsequent, especially long-term and psychosocial, complications of surgical interventions in breast cancer patients [1].

Axillary lymph node dissection (ALND), which is associated with a high incidence of serious postoperative complications (lymphedema, sensory disturbances, and limited upper extremity range-of-motion) [2], has been gradually replaced by sentinel lymph node biopsy (SLNB) in early-stage breast cancer and has become the standard in axillary staging [1]. Although indications for SLNB continue to expand (e.g., following neoadjuvant therapy), axillary dissection is still indicated in patients with greater axillary node involvement. These patients are then at risk of developing serious postoperative complications, especially - lymphedema of the upper extremity.

The risks of developing postoperative lymphedema are well known: the extent of surgical intervention in the axilla, high body-mass index, metastatic involvement of the axillary nodes, and subsequent radiotherapy of the axilla [3]. Of the risks mentioned above, only the extent of surgical intervention can be modified in the treatment of breast cancer.

Two particular options for preventing lymphedema have recently been investigated in breast cancer surgery by reducing the radicality of ALND. These options are axillary reverse mapping (ARM) $[4,5]$ and targeted axillary dissection (TAD) [6]. The TAD technique was developed to reduce the false-negative rate of SLN after neoadjuvant treatment in the clinically-positive axilla, thus reducing the rate of lymphedema indirectly.

TAD involves performing targeted extirpation of preoperatively-labeled lymph nodes (identified as infiltrated), rather than performing complete axillary dissection. For TAD to be safe and effective, the affected nodes must be safely identified and reliably labeled with a method that allows them to be identified at any time (graphite, seed, clip, wire), even following the completion of neoadjuvant chemotherapy; 
the nodes are then extirpated. The concept assumes that leaving other (unaffected) axillary nodes in situ will preserve drainage of the upper extremity and reduce the risk of developing lymphedema [7].

In ARM, the lymphatics that traverse the axilla and have been identified to drain the upper extremity are left in situ in axillary dissection. The concept is based on the assumption that the lymphatic drainage of the breast and upper extremity is functionally separate, at least as they pass through the axilla. Targeted dissection of breast lymphatics (and preservation of upper extremity lymphatics) in the axilla may thus reduce the incidence of upper extremity lymphedema.

The aim of the present study was to map the lymphatic drainage of the upper extremity that traverses the axilla and elucidate its relationship with the lymphatic drainage of the breast.

\section{Patients and methods}

Study design. The present study was conducted in 79 patients with advanced breast cancer in whom axillary lymph node dissection was indicated. The average age was 49 years (range 29-76 years, median 46 years). Indication to perform ALND was clinical verification of axillary node infiltration (category cN1, cN2), - based on palpation or imaging examinations. Prior to surgery, 37 patients underwent neoadjuvant chemotherapy, 28 patients received neoadjuvant hormonal therapy, and 14 were indicated for primary axillary dissection. Other parameters (e.g., biological characteristics of the tumor, type, and regimen of neoadjuvant chemotherapy, etc.) were not evaluated due to the limited sample size and large variability.

Lymph node visualization. Technetium-99m (99mTc) radionuclide nanocolloid (particle size $<80 \mathrm{~nm}$ ) was used to visualize the upper extremity lymphatics. Radionuclide administration was performed at two injection sites between the second and third metacarpophalangeal joints with a total activity of $80-100 \mathrm{MBq} 2-4$ hours prior to surgery. The aim of the application was to visualize as many upper extremity lymph nodes localized in the axilla as possible. We did not apply patent blue due to the potentially prolonged skin discoloration.

Surgical treatment. Patients were operated on in a supine position with the arm - secured at a 110-degree angle to their body. The axilla was defined by anatomical structures as follows: medially by the lateral chest wall, laterally by the lateral edge of the latissimus dorsi muscle, and cranially by the axillary vein. Previous work has shown that lymphatic vessels from the upper extremity extend parallel along, and up to $2 \mathrm{~cm}$ caudal to the axillary vein, approximately equivalent to the course of the $2^{\text {nd }}$ intercostobrachial nerve [8]. Therefore, attention was focused primarily on the part of the axilla inferior to the $2^{\text {nd }}$ intercostobrachial nerve, which also includes the central axillary region. During the operation, the axilla was divided into 6 quadrants according to obvious landmarks; the course of the thoracodorsal bundle divided the entire axilla into medial and lateral parts. These halves were further divided by the course of the $2^{\text {nd }}$ and $3^{\text {rd }}$ intercostobrachial nerves (in the case of a forked course, their cranial branch) into a total of 6 fields. Field no. I. also contained tissue from the apex of the axilla and subpectoral space (axillary level III. - see Figure 1). The traditional surgical procedure was modified so that it was possible to precisely identify individual groups of nodes and to avoid prolonging the operation time.

A C-Trak ${ }^{\infty}$ device was used for the intraoperative detection of radioactivity. The probe was orientated perpendicular to the operating table when detecting activity. Active lymph nodes superior to the axillary vein were left in situ. After verifying activity, the nodes were gradually resected and their position was recorded in a diagram. Active nodes were separated ex vivo and sent separately according to topographic localization for microscopic examination. All affected nodes (both macro- and micro-metastases) were recorded as positive. Neither tumor characteristics (size, grading, type, biological characteristics) nor the type and effect of neoadjuvant treatment were analyzed. The localization, involvement, radioactivity, and number of lymph nodes obtained were analyzed. Lymph nodes that were simultaneously metastatically affected and radioactive were the most interesting, confirming that lymph from both the breast and the upper extremity pass through these nodes. All surgeries were performed by a single surgeon.

The study protocol was approved by the local ethics committee and met the guidelines of the First Faculty of Medicine, Charles University in Prague, and General University Hospital. Informed consent was obtained from all subjects.

\section{Results}

In total, 1,109 lymph nodes were resected and examined. Complete pathological remission in the axilla after neoadjuvant chemotherapy was demonstrated in 8 cases. Radioactive nodes were found in all 79 patients. A total of 230 radioactive nodes were detected, i.e., an average of 2.9 radioactive nodes per subject. The mean number of resected lymph nodes per subject was 14 . A total of 195 metastatic lymph nodes were found. The mean number of metastatically affected nodes per subject was 2.4. A total of 21 nodes were resected that were both radioactive and metastatically affected. The distribution of lymph node groups resected in individual quadrants is shown in Table 1 and Figure 1.

Radioactivity in the axilla was detected in all patients. Even with assumed error in the topographical scheme, it was clear that part of the lymph (with tracer) from the upper extremity passes through the central and caudal axilla. In considering all 230 radioactive nodes, the graphical representation of lymph flow (tracer) according to the number and localization of radioactive nodes was as follows: $65 \%$ of lymph 
flowed along the axillary vein, $31 \%$ to the central axilla, and in $4 \%$ of cases to the caudal part of the axilla (Figure 2.). In 7 cases, activity was also detected in the periclavicular area. In no case was the activity recorded in the axillary part of the mammary gland. No adverse effects of the procedure (administration of tracer, allergic reaction, pain at the injection site) were recorded.

\section{Discussion}

The quality and success of lymphatic mapping depend on the physical properties of the substances used as contrast (particle size, solubility) and the time between application and operation [9]. When using larger-sized substances (sentiscint), the situation is reversed and a single node is displayed that accumulates activity and slowly releases the contrast further.

When using a nanocolloid with smaller particle size, one or more lymph nodes rapidly appear (in 1 hour), however, the substance rapidly passes to other nodes and the activity resolves relatively quickly (clearance). The patient's constitution also plays an important role in the absorption and passage of contrast. Patients with a higher body mass index experience a slower passage of the contrast and an increasing number of mapping failures [10]. Thus, it is difficult to determine the most suitable contrast agent and application timing to visualize as many nodes as possible. For ARM, it is preferable to use a method of lymphatic mapping that shows as many upper extremity nodes for as long as possible.

Despite careful design and tissue dissection in the present study, some inconsistencies may be assumed. The anatomical delineation of the axilla is very approximate, a challenge that many anatomists have faced due to the changes in relative proportions during upper extremity movement; small changes in upper extremity position can significantly change topographic conditions. Interindividual anatomical variability and constitution of the patient also play an important role. Despite these unfavorable factors, it is clear that a portion of the lymph from the upper extremity with contrast (tracer) passed through the central axilla, and potentially the caudal axilla. The same conclusion was reached by Ponzone et al. [11], who analyzed axillary nodules in 49 patients with breast cancer. They identified metastatic nodes in patients with the extensive nodal disease during ARM mapping,

Table 1. Distribution of resected lymph node groups in individual quadrants.

\begin{tabular}{lcccccc}
\hline Field & I & II & III & IV & V & VI \\
\hline Total number of nodes & 174 & 131 & 225 & 175 & 268 & 136 \\
Normal nodes & 83 & 44 & 151 & 107 & 164 & 114 \\
Radioactive nodes & 63 & 87 & 26 & 44 & 6 & 4 \\
Metastatic nodes & 22 & 0 & 42 & 17 & 96 & 18 \\
Radioactive and metastatic nodes & 6 & 0 & 6 & 7 & 2 & 0 \\
\hline
\end{tabular}

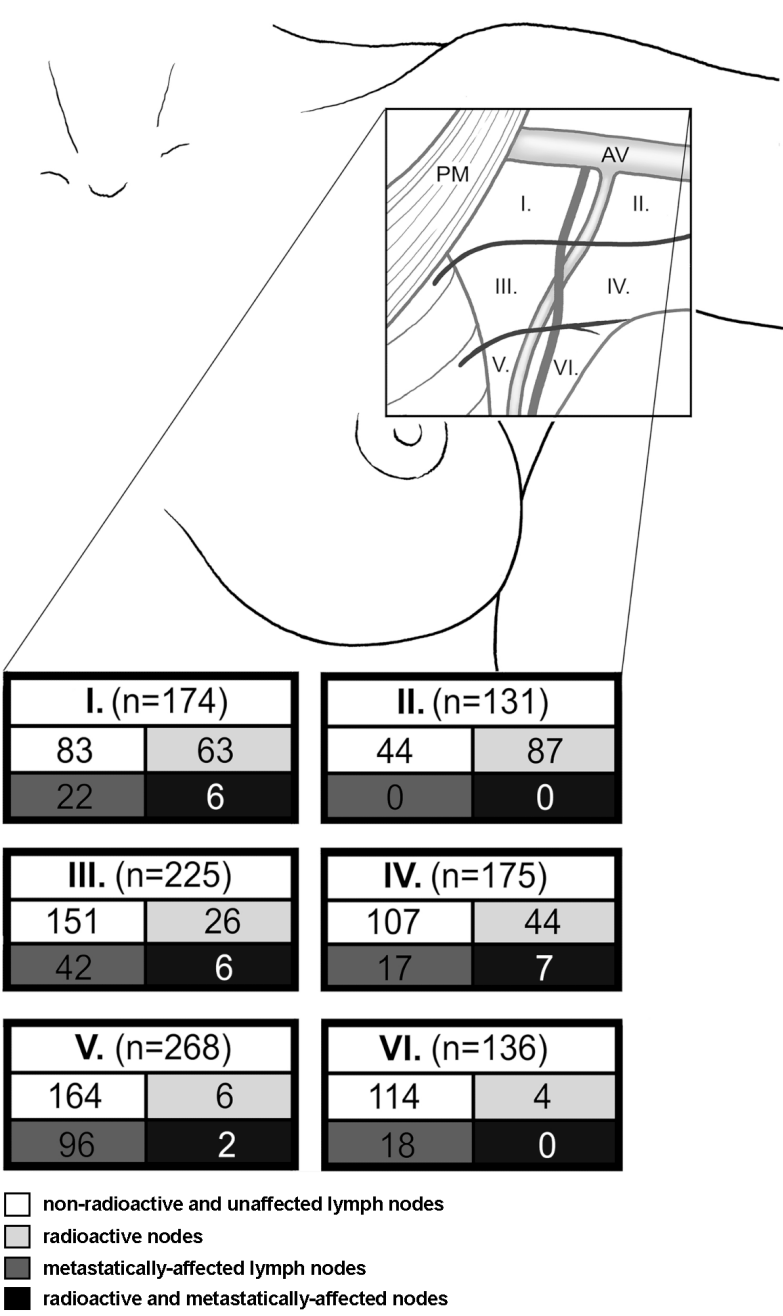

Figure 1. The number of physiological, radioactive, and metastaticallyaffected nodes in individual fields. Abbreviations: PM-pectoralis muscle; AV-axillary vein; AA-Apex of Axilla; ULA-Upper Lateral Axilla. I-IVarea of the axilla.

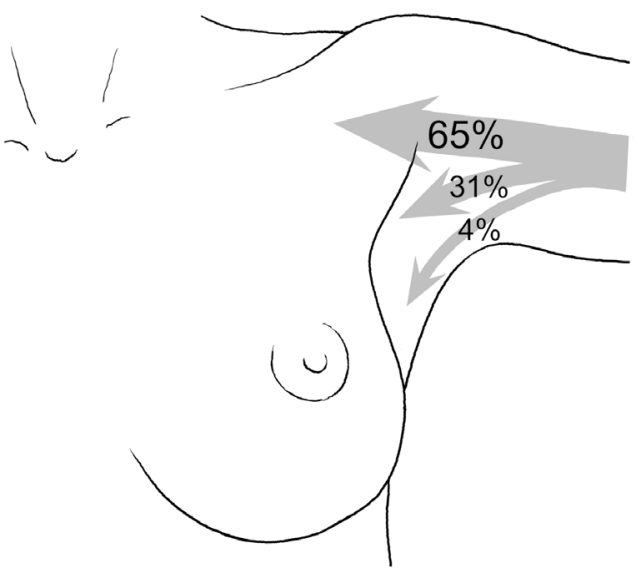

Figure 2. Schematic representation of the direction of lymph flow from the upper limb and its distribution in the axilla. Detailed explanation in the text. 
which demonstrated that there is no reliable separation of the upper extremity and breast lymphatic pathways.

Our findings may also explain the emergence of objectively measurable lymphedema in patients after SLNB, which De Groef et al. referenced [12]. The same assumption applies to TAD as well, in which only markedly affected metastatic lymph nodes are removed (sentinel and parasentinel nodes are primarily affected by metastases). The extent of lymphatic damage in TAD is greater than in SLNB, but likely does not affect the severity of lymphedema, as Goldberg et al. did not detect any dependence of lymphedema grade on the number of sentinel nodes resected [13].

In many previous studies, only the ARM node (sentinel node of the upper extremity) is shown after contrast application (primarily to the medial side of the upper extremity), and the contrast does not spread further. Despite the extensive application of contrast (patent blue) to other ARM nodes [7], other nodes are very difficult to visualize. Our application protocol (the tracer used and the site of application between the $2^{\text {nd }}$ and $3^{\text {rd }}$ metacarpophalangeal joints) likely made it possible to fully utilize a greater part of the upper extremity lymphatics than when contrast applied to the proximal inner arm. The identification of upper extremity-draining nodes in the axilla was successful in all patients, which is consistent with results reported in a systematic review by Beek et al. [14], in which ARM nodes were identified in $47-100 \%$ of patients who underwent ALND.

The most important finding in the present study was that 21 nodes were detected that were both radioactive and metastatically affected. This suggests that the lymph flows from both the breast and the upper extremity simultaneously to these nodes. Our results show that the axillary nodes have functional connections that form a network between them. Contrast media, therefore, does not flow only in a central direction to superior nodes, but on the contrary, these connections can reach all the axillary nodes including the sentinel nodes of the breast. This point is also consistent with the findings of Bedrosian et al. [8] and Nos et al. [15]. Similar findings were reported by Boneti et al. [16], who described the frequent finding of a blue-colored collector-close the sentinel lymph node at biopsy following the application of patent blue in the upper extremity. The same holds true for the study by Ma et al., which used a fluorescent tracer [17]. These connections may represent a potential pathway for metastatic cancer cells in sentinel node-positive patients. There are two possible explanations for the metastatic involvement of lymph nodes draining the upper extremity [18]. First, it could be a consequence of disease progression, as tumor growth may alter the pattern of lymphatic flow between the upper extremity and breast. Second, the anastomosis between the breast and upper extremity lymph nodes may also exist naturally, not only in the infraclavicular part but also in the central or caudal part of the axilla (Berg's level I or II) [19].

The American College of Surgeons Oncology Group Z107122 Alliance trial [20] and the SENTinel Neoadjuvant trial [21] found that the risk of metastases in ARM nodes was not significantly lower in patients who had received NAC when compared to those who had not. Our findings are consistent with those results.

Therefore, preserving the ARM lymph nodes may increase the risk of cancer recurrence, especially in clinically nodepositive patients [22].

Although initial studies on the oncological safety of ARM have been published [23], more data on lymphatic drainage and the ARM concept of the axilla are needed.

The results of the present study demonstrate may provide a new perspective on the concepts of minimally invasive procedures in the axilla (SLNB, TAD, ARM). Our results also explain the possible mechanical cause of upper extremity lymphedema after sentinel lymph node biopsy and in targeted axillary dissection.

In concept, ARM raises the question of potential oncological hazard when finding metastatically affected radioactive nodes (after application of radiopharmaceutical to the upper extremity).

We still have no clear understanding of the lymphatic drainage system of the upper extremity and breast. Our results show that part of the lymph from the upper extremity flows through nodes in the central part of the axilla and mixes with the lymph from the breast. This suggests that the lymphatic drainage of the upper extremity cannot be functionally separated from the lymphatic drainage of the breast. Our results also explain the possible mechanical cause of upper extremity lymphedema after sentinel lymph node biopsy. It now appears that ARM represents a way to improve rather than minimize surgery.

Acknowledgments: I would like to thank the academic painter Jan Kacvický for his assistance with graphic design. This work was supported by the Charles University in Prague (UNCE 204024 and PROGRES Q28/LF1).

\section{References}

[1] LYMAN GH, SOMERFIELD MR, BOSSERMAN LD, PERKINS CL, WEAVER DL et al. Sentinel Lymph Node Biopsy for Patients with Early-Stage Breast Cancer: American Society of Clinical Oncology Clinical Practice Guideline Update. J Clin Oncol 2017; 35: 561-564. https://doi.org/10.1200/ JCO.2016.71.0947

[2] WERNICKE AG, SHAMIS M, SIDHU KK, TURNER BC, GOLTSER Y et al. Complication rates in patients with negative axillary nodes 10 years after local breast radiotherapy after either sentinel lymph node dissection or axillary clearance. Am J Clin Oncol 2013; 36: 12-19. https://doi. org/10.1097/COC.0b013e3182354bda

[3] KHAN SA. Axillary reverse mapping to prevent lymphedema after breast cancer surgery: defining the limits of the concept. J Clin Oncol 2009; 27: 5494-5496. https://doi.org/10.1200/ JCO.2009.24.3311 
[4] KLIMBERG S. A new concept toward the prevention of lymphedema: axillary reverse mapping. J Surg Oncol 2008; 97: 563-564. https://doi.org/10.1002/jso.20905

[5] ABDELHAMID MI, BARI AA, FARID M, NOUR H. Evaluation of axillary reverse mapping (ARM) in clinically axillary node negative breast cancer patients - Randomised controlled trial. Int J Surg 2020; 75: 174-178. https://doi. org/10.1016/j.ijsu.2020.01.152

[6] SIMONS JM, VAN NIJNATTEN TJA, VAN DER POL CC, LUITEN EJT, KOPPERT LB et al. Diagnostic Accuracy of Different Surgical Procedures for Axillary Staging After Neoadjuvant Systemic Therapy in Node-positive Breast Cancer: A Systematic Review and Meta-analysis. Ann Surg 2019; 269: 432-442. https://doi.org/10.1097/SLA.0000000000003075

[7] DONKER M, STRAVER ME, WESSELING J, LOO CE, SCHOT $\mathrm{M}$ et al. Marking axillary lymph nodes with radioactive iodine seeds for axillary staging after neoadjuvant systemic treatment in breast cancer patients: the MARI procedure. Ann Surg 2015; 261: 378-382. https://doi.org/10.1097/ SLA.0000000000000558

[8] BEDROSIAN I, BABIERA GV, MITTENDORF EA, KUERER HM, PANTOJA L et al. A phase I study to assess the feasibility and oncologic safety of axillary reverse mapping in breast cancer patients. Cancer 2010; 116: 2543-2548. https:// doi.org/10.1002/cncr.25096

[9] SURASI DS, O'MALLEY J, BHAMBHVANI P. 99mTc-Tilmanocept: A Novel Molecular Agent for Lymphatic Mapping and Sentinel Lymph Node Localization. J Nucl Med Technol 2015; 43: 87-91. https://doi.org/10.2967/jnmt.115.155960

[10] DEROSSIS AM, FEY JV, CODY HS $3^{\text {RD }}$, BORGEN PI. Obesity influences outcome of sentinel lymph node biopsy in early-stage breast cancer. J Am Coll Surg 2003; 197: 896-901. https://doi.org/10.1016/j.jamcollsurg.2003.08.005

[11] PONZONE R, CONT NT, MAGGIOROTTO F, CASSINA E, MININANNI $P$ et al. Extensive nodal disease may impair axillary reverse mapping in patients with breast cancer. J Clin Oncol 2009; 27: 5547-5551. https://doi.org/10.1200/ JCO.2009.22.1846

[12] DE GROEF A, VAN KAMPEN M, TIETO E, SCHÖNWEGER P, CHRISTIAENS MR et al. Arm lymphoedema and upper limb impairments in sentinel node-negative breast cancer patients: A one year follow-up study. Breast 2016; 29: 102-108. https://doi.org/10.1016/j.breast.2016.07.021

[13] GOLDBERG JI, WIECHMANN LI, RIEDEL ER, MORROW M, VAN ZEE KJ. Morbidity of sentinel node biopsy in breast cancer: the relationship between the number of excised lymph nodes and lymphedema. Ann Surg Oncol 2010; 17: 3278-3286. https://doi.org/10.1245/s10434-010-1155-4
[14] BEEK MA, GOBARDHAN PD, SCHOENMAECKERS EJ. Axillary reverse mapping in axillary surgery for breast cancer: an update of the current status. Breast Canc Res Treat 2016; 158: 421-432. https://doi.org/10.1007/s10549-016$3920-\mathrm{y}$

[15] NOS C, KAUFMANN G, CLOUGH KB, COLLIGNON MA, ZERBIB E et al. Combined axillary reverse mapping (ARM) technique for breast cancer patients requiring axillary dissection. Aann Surg Oncol 2008; 15: 2550-2555. https://doi. org/10.1245/s10434-008-0030-z

[16] BONETI C, KOROURIAN S, DIAZ Z, SANTIAGO C, MUMFORD $S$ et al. Scientific Impact Award: Axillary reverse mapping (ARM) to identify and protect lymphatics draining the arm during axillary lymphadenectomy. Am J Surg 2009; 198: 482-487. https://doi.org/10.1016/j.amjsurg.2009.06.008

[17] MA X, WEN S, LIU B, LI D, WANG X et al. Relationship between Upper Extremity Lymphatic Drainage and Sentinel Lymph Nodes in Patients with Breast Cancer. J Oncol 2019; 2019: 8637895. https://doi.org/10.1155/2019/8637895

[18] NOGUCHI M, YOKOI M, NAKANO Y. Axillary reverse mapping for breast cancer. Breast Canc Res Treat 2010; 119: 529-535. https://doi.org/10.1007/s10549-009-0578-8

[19] HAMA Y, KOYAMA Y, URANO Y. Simultaneous two-color spectral fluorescence lymphangiography with near infrared quantum dots to map two lymphatic flows from the breast and the upper extremity. Breast Canc Res Treat 2007; 103: 23-28. https://doi.org/10.1007/s10549-006-9347-0

[20] BOUGHEY JC, SUMAN VJ, MITTENDORF EA. Sentinel lymph node surgery after neoadjuvant chemotherapy in patients with node-positive breast cancer: the ACOSOG Z1071 (Alliance) clinical trial. J Am Med Assoc 2013; 310: 1455-1461. https://doi.org/10.1001/jama.2013.278932[21] KUEHN T, BAUERFEIND I, FEHM T. Sentinel-lymph-node biopsy in patients with breast cancer before and after neoadjuvant chemotherapy (SENTINA): a prospective, multicentre cohort study. Lancet Oncol 2013; 14: 609-618. https:// doi.org/10.1016/S1470-2045(13)70166-9

[22] SHAO X, SUN B, SHEN Y. Axillary reverse mapping (ARM): where to go. Breast Cancer 2019; 26: 1-10. https://doi. org/10.1007/s12282-018-0886-0

[23] GENNARO M, LISTORTI C, MARIANI L, MACCAURO $\mathrm{M}, \mathrm{BIANCHI} \mathrm{G}$ et al. Oncological safety of selective axillary dissection after axillary reverse mapping in node-positive breast cancer. Eur J Surg Oncol 2021; 47: 1606-1610. https:// doi.org/10.1016/j.ejso.2020.10.031 